Canad. J. Math. Vol. 53 (4), 2001 pp. 696-714

\title{
Avoiding Patterns in the Abelian Sense
}

\author{
J. Currie and V. Linek
}

Abstract. We classify all 3 letter patterns that are avoidable in the abelian sense. A short list of four letter patterns for which abelian avoidance is undecided is given. Using a generalization of Zimin words we deduce some properties of $\omega$-words avoiding these patterns.

\section{Introduction}

The study of words avoiding certain patterns is an area of combinatorics on words reaching back to at least the turn of the century and the work of Thue. A word in which no two adjacent subwords are identical is called square-free; thus a square-free word $w$ can never be written $w=x y y z$ with $y$ a non-empty word. As an example, orange is square-free, but banana is not, since banana $=b$ an an $a$ contains an twice in a row.

One quickly checks that the longest square-free words over the alphabet $\{a, b\}$ are $a b a$ and $b a b$; no word of length 4 or more over a 2 letter alphabet is squarefree. Nevertheless, in 1906 Thue [17] showed that over a 3 letter alphabet, there are arbitrarily long square-free words. By König's Tree Lemma, this is equivalent to saying that there is an infinite square-free sequence on 3 letters. A word in which no three adjacent subwords are identical is called cube-free. Thue also showed that there is an infinite cube-free sequence over a 2 letter alphabet. A very readable modern account of Thue's work can be found in [3].

Thue's discoveries have numerous algebraic applications. One such is to the Burnside problem for groups. The solution of this problem by Novikov and Adjan [14] employed a cube-free sequence at a key point. More recently, square-free sequences have been used to study equational classes of semigroups [4] and non-finitely based varieties [16]. In this algebraic context, it becomes natural to consider not only square-free and cube-free words, but words avoiding other patterns.

Via a construction credited to Dilworth by Morse [13], the existence of an infinite square-free sequence on 3 symbols is equivalent to the existence of an infinite semigroup with 3 generators, obeying the law $x^{2}=e$. Similarly, the existence of an infinite cube-free sequence on 2 symbols is equivalent to the existence of an infinite semigroup which obeys the law $x^{3}=e$, and has 2 generators. In the study of semigroup varieties, the following Burnside type question is natural:

Given an arbitrary word $p$ in one or more variables, is there a finitely generated infinite semigroup satisfying the law $p=e$ ?

Received by the editors September 25, 1999; revised March 8, 2001.

Research of both authors supported by NSERC Operating Grants.

AMS subject classification: 05,68 .

(c) Canadian Mathematical Society 2001. 


\begin{tabular}{|ll|c|}
\hline \multicolumn{1}{|c|}{ Pattern $p$} & $\begin{array}{c}\text { Smallest } k \text { such that } \\
p \text { is } k \text {-avoidable }\end{array}$ \\
\hline$y$ & $\infty$ \\
$y^{2}$ & $(=y y)$ & 3 \\
$y^{n}$, & $n \geq 3$ & 2 \\
\hline
\end{tabular}

Table 1: The $k$-avoidability of patterns over a one letter alphabet.

Let $\Sigma$ be a finite alphabet, and let $\Sigma^{*}$ be the free monoid over $\Sigma$. Thus $\Sigma^{*}$ consists of all finite strings of symbols in $\Sigma$, called words, with the operation of concatenation. The word in $\Sigma^{*}$ with no letters, the empty word, is denoted $\epsilon$. The length of word $w$ is the number of letters in it, denoted by $|w|$. Thus $\mid$ banana $|=6,| \epsilon \mid=0$. We will also consider infinite one-way words over $\Sigma$ of the form $w=w_{1} w_{2} w_{3} \cdots$, which we call $\omega$-words. The set of $\omega$-words over $\Sigma$ is denoted by $\Sigma^{\omega}$. Words are finite unless otherwise indicated. If $p=p_{1} p_{2} \cdots p_{k}$ is a string and $w$ is a finite or infinite word then $w$ encounters $p$ if there are words $t, v, X_{i}$ such that $w=t X_{1} X_{2} \cdots X_{k} v$, each $X_{i}$ is non-empty and $X_{i}=X_{j}$ whenever $p_{i}=p_{j}$. Equivalently, we say $p$ occurs in $w$. We call $p$ a pattern and write $w \vdash p$ if pattern $p$ occurs in $w$. If $p$ does not occur in $w$ then we write $w \forall p$ and say that $w$ avoids $p$. Thus 'banana' encounters $y y$, while 'orange' avoids $y y$.

If arbitrarily long words avoiding $p$ exist over an alphabet of size $k$, then $p$ is $k$ avoidable, otherwise $p$ is $k$-unavoidable. By König's Tree Lemma, $p$ is $k$-avoidable if and only if there is an $\omega$-word on $k$ letters that avoids $p$.

Thue's work shows that $y y$ and $y y y$ are avoidable. In fact, everything about avoiding patterns on one letter has been known since 1906. (See Table 1.)

While some patterns are avoidable, others are unavoidable; consider $x y x$. If $w$ is a long enough word over a fixed finite alphabet, then $w$ has a repeated letter, say $u$. Word $w$ will thus have a subword ${ }^{1} u v u$, some word $v$, and $u v u$ is a coded version of xyx.

A characterization of avoidable patterns has been given by Zimin [18], and independently by Bean et al. [2]. According to Zimin, pattern $p$ over an alphabet of $n$ letters is avoidable if $p$ is avoided by $Z_{n}$, where $Z_{n}$ is defined recursively by

$$
Z_{n}= \begin{cases}1, & n=1 \\ Z_{n-1} n Z_{n-1}, & n>1\end{cases}
$$

The following problem has been open for 20 years [2]:

Find an algorithm to decide, given an avoidable pattern $p$, the smallest $k$ such that $p$ is $k$-avoidable.

Progress has been made on this problem given restrictions on $p$. For example, Cassaigne [6] solved the problem when $p$ is a pattern over a 2 letter alphabet. Cassaigne also gives an inventory of the state of knowledge when $p$ is a pattern over a

\footnotetext{
${ }^{1}$ In this context, a subword of a word is always contiguous: ana is a subword (2 ways!) of banana, but $a a$ is not. The opposite of a subword is an extension: banana is an extension of ana.
} 


\begin{tabular}{|ll|c|}
\hline Pattern $p$ & $\begin{array}{c}\text { Smallest } k \text { such that } \\
p \text { is } k \text {-avoidable } \\
\text { in the abelian sense }\end{array}$ \\
\hline$y$ & $\infty$ \\
$y^{2}$ & $(=y y)$ & 4 \\
$y^{3}$ & & 3 \\
$y^{n}$, & $n \geq 4$ & 2 \\
\hline
\end{tabular}

Table 2: The $k$-avoidability in the abelian sense of $y^{n}$.

3 letter alphabet. For example, abcbabc is known to be 3 -avoidable, but perhaps is 2-avoidable.

In algebraic problems, commutativity is usually a simplifying assumption. In 1961, Erdős [10] asked whether an infinite sequence over a finite alphabet existed which was non-repetitive in an abelian sense; no 2 adjacent blocks were to be permutations of one another. Not only 12341234 is forbidden in such a sequence, but also 12342134 , since 1234 and 2134 are permutations of each other.

A sequence avoiding $y y$ in this abelian sense was discovered by Evdokimov [11], but he used an alphabet of 25 letters. The alphabet size was reduced to 5 letters by Pleasants [15], and not until 1992, to a 4 letter alphabet by Keränen [12]. One checks that on a 3 letter alphabet there are only finite sequences which are non-repetitive in this abelian sense. On the other hand, Dekking [9] showed that the smallest alphabet on which $y^{3}$ was avoidable in this abelian sense had 3 symbols, while 2 symbols were necessary and sufficient to avoid $y^{4}$ in the abelian sense. We see then, that our understanding of avoiding patterns in the abelian sense is only now at the same level that our understanding of ordinary pattern avoidance was in 1906!

If $X$ and $Y$ are words then we say that $X \sim Y$ if and only if the letters of $X$ are a permutation of the letters of $Y$. In words, $X$ is abelian equivalent to $Y$. If $p=$ $p_{1} p_{2} \cdots p_{k}$ is a string and $w$ is a finite or infinite word then $w$ encounters $p$ in the abelian sense if $w=t X_{1} X_{2} \cdots X_{k} v$, each $X_{i}$ is non-empty and $X_{i} \sim X_{j}$ whenever $p_{i}=p_{j}$. Equivalently, we say $p$ occurs in $w$ in the abelian sense. We write $w \models p$ if $w$ encounters $p$ in the abelian sense, $w \not \models p$ otherwise. If an $\omega$-word avoiding $p$ in the abelian sense exists over an alphabet of size $k$, then $p$ is $k$-avoidable in the abelian sense, otherwise $p$ is $k$-unavoidable in the abelian sense.

From now on we have a distinction between ' $w$ encounters $p$ ', and ' $w$ encounters $p$ in the abelian sense', etc. In the first case we say by way of emphasis that ' $w$ encounters $p$ in the ordinary sense'. Suppose that $P$ is a set of patterns. We extend our definitions, saying $P$ is $k$-avoidable ( $k$-avoidable in the abelian sense) if there is an $\omega$-word $w$ on a $k$-letter alphabet such that $w \not \forall p(w \not \models p)$ for all $p \in P$. We write $w \not \forall P(w \not \models P)$. Let $S$ be a set of words. We say that $w$ avoids subwords in $S$ if no word of $S$ is a subword of $w$.

Knowledge of abelian avoidability is in a very primitive state. It is not even known which patterns are abelian avoidable. We show (Lemma 3 ) that avoidability in the abelian sense is not the same as avoidability in the ordinary sense. In Section 3.1 
we completely determine which patterns over a 3 letter alphabet are avoidable in the abelian sense.

Theorem 1 A pattern $p$ on 3 letters is avoidable in the abelian sense if and only if $Z_{3} \forall p$.

To have the same result on 4 letters (see Section 3.2), it would suffice to show that the following six patterns are avoidable in the abelian sense.

$\begin{array}{ll}b_{1} & 01020312 \\ b_{2} & 01020321 \\ b_{3} & 01021303 \\ b_{4} & 01023031 \\ b_{5} & 010203013 \\ b_{6} & 010213020\end{array}$

For these 6 patterns there are considerable restrictions on an $\omega$-word avoiding any of them. For example, none of these patterns is 4 -avoidable in the abelian sense. If one of these patterns turns out to be avoidable in the abelian sense, then we have another contrast with ordinary avoidance, where no pattern is known to be 5-avoidable, but not 4-avoidable [1], [8].

The following conjecture seems natural:

Conjecture 2 A pattern $p$ on $n$ letters is avoidable in the abelian sense if and only if $Z_{n} \not=p$.

\section{Avoidable and Unavoidable Patterns}

To begin with, we establish that avoidability in the abelian sense is not the same as avoidability in the ordinary sense.

Lemma 3 The pattern $p=$ abcabadabacba is avoidable in the ordinary sense, but not avoidable in the abelian sense.

Proof By Zimin's criterion, to show that $p$ is avoidable it is enough to show that $p$ does not occur in $Z_{4}=121312141213121$ in the ordinary sense. Suppose on the contrary that $Z_{4}$ has a subword $P=A B C A B A D A B A C B A$ where $A, B, C$ and $D$ are non-empty words. Then $6|A|+4|B|+2|C|+|D|=|P| \leq\left|Z_{4}\right|=15$, whence $|A|=|B|=1$. By considering the frequencies of letters in $P$ and $Z_{4}$ we see that $A=1$ and $B=2$. We see that the first and second occurrences of $A B$ in $P$ must correspond to the first and second occurrences of 12 in $Z_{4}$, hence $C=13$. On the other hand by considering the last and the next to last occurrences of $B A$ in $P$, we deduce that $C=31$, a contradiction. This shows that $p$ is avoidable in the ordinary sense. On the other hand, the parsing $Z_{4}=1,2,13,1,2,1,4,1,2,1,31,2,1$ shows that $p$ occurs in $Z_{4}$ in the abelian sense, which shows that $p$ is unavoidable in the abelian sense, as $Z_{4}$ is unavoidable. 


\subsection{Avoidable Patterns}

A fractional abelian spower is a word of the form $u v w$ where $w \sim u$, and $|u v w| /|u v|=$ s. For example, madam $=$ ma $d$ am is a fractional abelian $5 / 3$ power. We allow $v$ to be empty in our definition, so it is correct to say that teammate = team mate is a fractional abelian 2 power, although we prefer to call it an abelian square. Also allowable is $u=w=\epsilon$, so it is also correct to say that orange is a fractional abelian 1 power. When $u, v$ and $w$ are all empty the ratio $|u v w| /|u v|$ is not defined, but it makes sense to say that $u v w=\epsilon$ is a fractional 0 power since $x^{0}=\epsilon$ in the free monoid, and this is the convention we will make.

We say that a word $w$ contains a $r^{+}$fractional abelian power if $w$ has a subword that is a fractional $s$ abelian power with $s \geq r$. The notation $w \models x^{r+}$ indicates that $w$ contains a fractional abelian $r^{+}$power. Otherwise $w$ avoids $x^{r+}$.

Theorem 4 ([7]) Let $r \in(1,2]$. There is an $\omega$-word $w$ over some finite alphabet such that $w$ avoids $x^{r+}$.

Call a word $w$ doubled if each letter of $w$ occurs at least twice in $w$.

Lemma 5 Let $p$ be a doubled pattern with $|p|=l$. Suppose $q \models p$. Then $q \models x^{r+}$ where $r=1+(l-1)^{-1}$.

Proof Let $p=a_{1} a_{2} \cdots a_{l}$ and suppose that $q=u A_{1} A_{2} \cdots A_{l} v$ where $A_{i} \neq \epsilon$ for each $i$ and $A_{i} \sim A_{j}$ whenever $a_{i}=a_{j}$. Let $M=\max \left\{\left|A_{k}\right|: 1 \leq k \leq l\right\}$. Since $p$ is doubled we may choose $i<j$ such that $a_{i}=a_{j}$ and $\left|A_{j}\right|=M$. Let $u=A_{i}, v=A_{i+1} \cdots A_{j-1}$, $w=A_{j}$. Then $u \sim w$ and

$$
\begin{aligned}
\frac{|u v w|}{|u v|} & =1+\frac{|w|}{|u v|} \\
& =1+\frac{\left|A_{j}\right|}{\left|A_{i} \cdots A_{j-1}\right|} \\
& \geq 1+\frac{\left|A_{j}\right|}{(j-i)\left|A_{j}\right|} \\
& \geq 1+\frac{1}{l-1} .
\end{aligned}
$$

Thus $q \models x^{r+}$, where $r=1+(l-1)^{-1}$.

Lemma 6 Any word $w$ over an $n$ letter alphabet with $|w| \geq 2^{n}$ contains a doubled subword.

Proof Let $l_{n}, n \geq 1$ be the length of the longest word on $n$ letters with no doubled subword. We shall prove by induction that $l_{n} \leq 2^{n}-1$. Clearly $l_{1}=1=2^{1}-1$. Suppose the inequality holds for $n$ and let $w$ be a $n+1$ letter word. If $w$ has no doubled subword then some letter, $a$ say, occurs only once in $w$ and $w=w_{1} a w_{2}$ where $w_{1}, w_{2}$ 
are words on at most $n$ letters. Neither of $w_{1}$ and $w_{2}$ can have a doubled subword since $w$ has no doubled subword. By induction, $\left|w_{1}\right| \leq 2^{n}-1$ and $\left|w_{2}\right| \leq 2^{n}-1$. Thus $|w| \leq 2\left(2^{n}-1\right)+1=2^{n+1}-1$, as desired.

Combining the last two lemmas and Theorem 4 gives us the following.

Lemma 7 Any pattern $p$ on $n$ letters with $|p| \geq 2^{n}$ is avoidable in the abelian sense.

We shall see that the avoidability of fractional abelian powers has further implications for the abelian avoidance of general patterns. Let $p$ be a pattern and let $r \in[1,2]$. We say that $p$ forces $x^{r+}$ if $w \models p$ implies $w \models x^{r+}$, and we write $p \rightsquigarrow x^{r+}$. If $p=\epsilon$ then $p$ does not force any positive powers, since $p$ may occur in the empty string and the empty string contains no positive powers (by our earlier convention). However, the empty string is just $x^{0}$, so certainly $\epsilon \rightsquigarrow x^{0+}$. If $p \neq \epsilon$ then trivially $p \rightsquigarrow x^{1+}$, and hence also $p \rightsquigarrow x^{0+}$. Having dealt with the degenerate cases let us now show that abacbab $\rightsquigarrow x^{3 / 2+}$.

\section{Lemma 8 The pattern abacbab forces $x^{3 / 2+}$.}

Proof Suppose $w=t A_{1} B_{1} A_{2} C B_{2} A_{3} B_{3} z$ where the $A_{i}, B_{i}$ and $C$ are non-empty words and $A_{1} \sim A_{2} \sim A_{3}, B_{1} \sim B_{2} \sim B_{3}$. If $\left|A_{1}\right| \geq\left|B_{1}\right|$, then $\left|A_{1} B_{1} A_{2}\right| /\left|A_{1} B_{1}\right| \geq 3 / 2$, so $w$ contains the $3 / 2+$ power $A_{1} B_{1} A_{2}$. Otherwise, $\left|B_{1}\right|>\left|A_{1}\right|$, and $w$ contains the $3 / 2+$ power $B_{2} A_{3} B_{3}$.

Let $w$ be an $\omega$-word avoiding $x^{3 / 2+}$ powers. Then $w$ avoids abacbab. This method of showing avoidability can be generalized:

Let $p=p_{1} p_{2} \cdots p_{n}$ be a pattern with letters $p_{i}$. Suppose that $p$ is unavoidable in the abelian sense. Fix $r \in(1,2]$, and let $w$ be an $\omega$-word avoiding $x^{r+}$. Since $p$ is unavoidable, let $P=P_{1} P_{2} \cdots P_{n}$ be a subword of $w$ where the $P_{i}$ are non-empty, and $P_{i} \sim P_{j}$ if $p_{i}=p_{j}$. Given $r$, and then $P$, define the $P$-length of a subword $T=p_{i} p_{i+1} \cdots p_{j}$ of $p$ to be $|T|_{P}=\left|P_{i}\right|+\left|P_{i+1}\right|+\cdots+\left|P_{j}\right|$. Suppose that $X Y Z$ is a subword of $p$, with $X \sim Z$. Since $w$ avoids $x^{r+}$, we must have $|X Y Z|_{P} /|X Y|_{P}<r$, or what is the same

$$
|Z|_{P}<(r-1)|X Y|_{P}
$$

From the pattern $p$ we then get a whole system of inequalities, one for each fractional power $X Y Z$ of $p$ with $X \sim Z$.

Now let $p$ be an arbitrary pattern, and let $r \in[1,2]$. For each distinct letter $p_{j}$ of $p$ introduce a variable, $l_{j}$, which stands for the length of a string corresponding to letter $p_{j}$ in an occurrence of $p$. For each subword $T=p_{i} p_{i+1} \cdots p_{j}$ of $p$ we define $l_{T}$ to be the sum $l_{p_{i}}+l_{p_{i+1}}+\cdots+l_{p_{j}}$. According to (1) above we create a system of inequalities $S(p, r)$ as follows:

$$
S(p, r):\left\{l_{Z}<(r-1) l_{X Y}: p=\alpha X Y Z \beta, X \sim Z\right\}
$$


In the case where $r \in(1,2]$ we exploit the fact that $l_{X}=l_{Z}$ and rewrite the system $S(p, r)$ in the equivalent form

$$
S(p, r):\left\{\left[(r-1)^{-1}-1\right] l_{X}<l_{Y}: p=\alpha X Y Z \beta, X \sim Z\right\}
$$

Henceforth it is tacitly understood that if $r=1$ the first form of the system is to be used, and the second, tidier form is to be used for all $r \in(1,2]$.

Clearly, if $r=1$ then system $S(p, r)$ is inconsistent, since the $l_{j}$ are integer variables. In the case that $p$ is unavoidable, we see that the system $S(p, r)$ is consistent for each $r \in(1,2]$ by taking $l_{p_{j}}=\left|p_{j}\right|_{P}$ for each letter $p_{j}$ of $p$ as above. In the case of the simplest avoidable pattern, $p=a a$, and the largest exponent, $r=2$, the system $S(a a, 2)$ defined above is just the inconsistency $0<0$, which shows that any $\omega$-word avoiding $x^{2+}$ powers automatically avoids $p$. More interesting is the case where $r \in(1,2)$. For example, consider again Lemma 8 , where $p=a b a c b a b$ and $r=3 / 2$. There $(r-1)^{-1}-1=1$ and the fractional abelian powers in $p$ are $a b a$, $a b a c b a, b a c b, b a c b a b, b a c b a, a c b a, a b a c b a b$ and $b a b$. The system $S(a b a c b a b, 3 / 2)$ is

$$
\begin{gathered}
l_{a}<l_{b} \\
l_{a}<l_{b}+l_{a}+l_{c}+l_{b} \\
l_{a}+l_{b}<l_{a}+l_{c} \\
l_{b}<l_{a}+l_{c} \\
l_{b}<l_{a}+l_{c}+l_{b}+l_{a} \\
l_{b}+l_{a}<l_{c}+l_{b} \\
l_{b}+l_{a}<l_{c} \\
l_{a}<l_{c}+l_{b} \\
l_{a}+l_{b}<l_{a}+l_{c}+l_{b} \\
l_{b}<l_{a} .
\end{gathered}
$$

One immediately notices that the last inequality is the inversion of the first inequality. This is a contradiction! We deduce that abacbab is avoidable, and in fact that $a b a c b a b \rightsquigarrow x^{3 / 2+}$, in accordance with Lemma 8 . When $S(p, r)$ is inconsistent we say that $p$ has an inversion. All nonempty patterns have an inversion for $r=1$, the trivial inversion. Typically when we speak of a pattern having an inversion we mean a nontrivial inversion with $r \in(1,2]$. Note that the inequalities involving the variable $l_{c}$ are of no use in deducing the inconsistency of $S(a b a c b a b, 3 / 2)$. This is because variable $l_{c}$ occurs only on the right hand side of the inequalities and it may be taken to be arbitrarily large. Indeed, for no value of $r \in(1,2]$ will the presence of the variable $l_{c}$ affect the consistency of the system, as we invite the reader to check. The fact that letter $c$ occurs just once in $p$ is what confines the singular appearances of variable $l_{c}$ to the right hand sides of the inequalities. It makes sense to delete the inequalities involving $l_{c}$, and we should like to effect this deletion by deleting the letter $c$ from abacbab. This motivates the discussion of the following paragraph. 
Let $p_{1}=a_{1} a_{2} \cdots a_{n}$ and $p_{2}=b_{1} b_{2} \cdots b_{m}$ be patterns with occurrences $w=$ $u A_{1} A_{2} \cdots A_{n} v \models p_{1}$ and $w=s B_{1} B_{2} \cdots B_{m} t \models p_{2}$. The occurrences of $p_{1}$ and $p_{2}$ are consistent if $A_{i} \sim B_{j}$ whenever $a_{i}=b_{j}$, and in that case we write $w \models p_{1-} p_{2}$. This leads to a generalization of the notion of a pattern, where we define an abelian occurence of $p=p_{1-} p_{2-} \cdots p_{n}$ in $w$ to consist of occurences of patterns $p_{1}, p_{2}, \ldots, p_{n}$ in $w$ that are mutually consistent in pairs. We say that $p_{1}, p_{2}, \ldots, p_{n}$ are the $n$ components of $p$, and that $p$ is connected if $n=1$ and disconnected if $n>1$.

If $p=p_{1-} p_{2-} \cdots p_{n}$ is a disconnected pattern and $r \in[1,2]$ we define $S(p, r)$ to be $S\left(p_{1}, r\right) \cup S\left(p_{2}, r\right) \cup \cdots \cup S\left(p_{n}, r\right)$. The next lemma should come as no surprise.

Lemma 9 Let $p \neq \epsilon$ be a connected or disconnected pattern, $c$ a letter of $p$ that occurs at most once in each component of $p$, and $p^{\prime}$ the pattern that results from $p$ upon deleting letter $c$. Then for any $r \in(1,2]$ the systems $S(p, r)$ and $S\left(p^{\prime}, r\right)$ are both consistent or both inconsistent.

Proof Let the system $S_{c}(p, r)$ consist of those inequalities in $S(p, r)$ that involve the variable $l_{c}$. Then $S(p, r)=S_{c}(p, r) \cup S\left(p^{\prime}, r\right)$. Clearly if $S\left(p^{\prime}, r\right)$ is inconsistent then $S(p, r)$ is inconsistent. If on the other hand the system $S\left(p^{\prime}, r\right)$ is consistent then we see that the variable $l_{c}$ occurs only on the right hand side of the inequalities of $S_{c}(p, r)$, and then exactly once in each inequality. This means that a given solution of the system $S\left(p^{\prime}, r\right)$ can be extended to a solution of $S(p, r)$ by taking $l_{c}$ sufficiently large. This shows that the two system are both consistent or both inconsistent. The proof is complete.

Even if Lemma 9 cannot be applied there are still simplifications that can be made. Consider the substring $a b a c b a$ of the pattern $p=a b a c b a b$. The two parsings $a b a c b a$ and $a b a c b a$ show that $a b a c b a$ is both a fractional $6 / 5$ and a fractional abelian $3 / 2$ power. These two powers yield the inequalities (3) and (4) in $S(a b a c b a b, 3 / 2)$ above. Clearly inequality (4) implies inequality (3), and this will still be the case if we vary $r \in(1,2]$, so naturally we delete the weaker inequality. In general let $p=\alpha X Y Z \beta=$ $\alpha^{\prime} X^{\prime} Y^{\prime} Z^{\prime} \beta^{\prime}$ with $X \sim Z$ and $X^{\prime} \sim Z^{\prime}$. For strings $s$ and $t$ we say that $s$ is lighter than $t$ if the multiset of letters of $s$ is strictly contained in the multiset of letters of $t$, and we write $s \sqsubset t$. For example, abac $\sqsubset$ cbaadcad, but abac $\not \subset$ caadcad. This gives a partial ordering of the substrings of $p$. We say that the fractional power $X Y Z$ supersedes the fractional power $X^{\prime} Y^{\prime} Z^{\prime}$ if $X^{\prime} \sqsubseteq X$ and $Y \sqsubseteq Y^{\prime}$, and at least one of $X^{\prime} \sqsubset X$ or $Y \sqsubset Y^{\prime}$ holds. We write $X^{\prime} Y^{\prime} Z^{\prime} \prec X Y Z$ if $X Y Z$ supersedes $X^{\prime} Y^{\prime} Z^{\prime}$. Finally, we prune $S(p, r)$ to the equivalent system

$S(p, r)^{\prime}:\left\{\left[(r-1)^{-1}-1\right] l_{X}<l_{Y}: p=\alpha X Y Z \beta, X \sim Z\right.$, and $X Y Z$ is $\prec$-maximal $\}$.

For example, the system $S\left(a b c b a_{-} c b a b c, 3 / 2\right)^{\prime}$ consists of just two inequalities, and is inconsistent. We define the inversive threshold, $r(p)$, of a pattern $p \neq \epsilon$ by the formula

$$
r(p)=\inf \left\{r \in[1,2]: S(p, r)^{\prime} \text { is inconsistent }\right\}
$$

and by convention we set $r(\epsilon)=0$. Note that $r(p)$ is defined for all patterns $p$, since $S(p, 1)$ is inconsistent for any $p \neq \epsilon$. Equivalently, for a nonempty pattern 
$p=p_{1-} p_{2}-\cdots-p_{n}$ we can define $r(p)$ as

$$
r(p)=\inf _{l} \max \left\{\frac{l_{X Y Z}}{l_{X Y}}: p_{k}=\alpha X Y Z \beta, X \sim Z, 1 \leq k \leq n\right\},
$$

where the infimum is taken over all functions $l$ that assign positive integer lengths to the letters of $p$. We now describe an algorithm based on Lemma 9 that gives a lower bound for $r(p)$.

Given a pattern, $p$, we let $m(p)$ denote the multiset of letters of $p$, so $m($ abacdabebaca $)=\{a, a, a, a, a, b, b, b, c, c, d, e\}$. Given a function, $l$, that assigns positive integer lengths to the letters of $p$ and two sub-multisets $U, V \subset m(w)$ we say that $U$ is longer than $V$ under $l$ if the sum of the lengths of the letters in $U$ is larger than the sum of the lengths of the letters in $V$. With $l: a \mapsto 1, b \mapsto 2$, $c \mapsto 3, d \mapsto 4, e \mapsto 5$ we have that $\{a, a, b, b\}$ is longer than $\{a, d\}$, but shorter than $\{a, c, c\}$. Given $p=\alpha X Y Z \beta, X \sim Z$, and a letter $j$ of $X$ consider all assignments $l$ with $l_{j} \geq l_{i}$ for all letters $i$ in $X Y Z$. In an occurrence of $p$ where $l$ gives the lengths of the strings corresponding to letters of $p$, what fractional power can we guarantee $X Y Z$ will correspond to? The answer is $(k+2) /(k+1)$, where $k \geq 1$ is the smallest integer for which there is a partition $m(Y)=Y_{1} \cup Y_{2} \cup \cdots \cup Y_{k}$ such that $m(X)$ is longer than each $m\left(Y_{i}\right)$ under $l$. Specifically, since all we know is that $l_{j}$ is largest among all $l_{i}$, this means that for $1 \leq m \leq k$ there are partitions $m(X)=J \cup O$ and $m\left(Y_{i}\right)=J^{\prime} \cup O^{\prime}$ such that $J$ consists of all the letters $j$ in $X, O^{\prime} \subseteq O$ and $|J| \geq\left|J^{\prime}\right|$. We write $R_{j}(X, Y, Z)=(k+2) /(k+1)$. For example, for $p$ above we have $R_{c}(a b a c$, dabe, baca $)=4 / 3$, since we have the partition $m($ dabe $)=\{a, b, d\} \cup\{e\}$, and no shorter partition will do. Similarly $R_{a}(a b a c$, dabe, baca $)=4 / 3$ and $R_{b}(a b a c$, dabe, baca $)=5 / 4$.

Given a non empty pattern $p=p_{1-} p_{2}-\cdots-p_{n}$ and a letter $j$ of $p$ define $r_{j}(p)$ by

$$
r_{j}(p)=\max \left\{R_{j}(X, Y, Z): p_{k}=\alpha X Y Z \beta, X \sim Z, 1 \leq k \leq n, \text { and } j \text { occurs in } X\right\} .
$$

Thus $r_{a}(a b a c a b c)=3 / 2, r_{b}(a b a c a b c)=5 / 4$ and $r_{c}(a b a c a b c)=4 / 3$. Continuing with this example, suppose that $w \models a b a c a b c$. If $l_{a}$ is the largest among $l_{a}, l_{b}, l_{c}$ then the string in $w$ corresponding to $a b a$ is a $3 / 2+$ power. Similarly, if $l_{b}$ is the largest then bacab corresponds to a string in $w$ that is a $5 / 4+$ power, and if $l_{c}$ is largest then $c a b c$ corresponds to a $4 / 3+$ power. We conclude that abacabc $\rightsquigarrow x^{5 / 4+}$, as $5 / 4$ is the minimum of $3 / 2,4 / 3$ and $5 / 4$. From this discussion it is clear that if we define

$$
\underline{r}(p)=\min \left\{r_{j}(p): j \text { a letter of } p\right\}
$$

then $p$ forces a $\underline{r}(p)+$ power. If we define $\bar{r}(p)$ to be the largest fractional abelian power in $p$ then this is clearly an upper bound for $r(p)$, since the infimum of equation 13 includes the function that assigns length 1 to each letter. We now have an algorithm for bounding $r(p)$ from below:

Theorem 10 Given a pattern $p=p_{1_{-}} p_{2_{-}} \cdots_{-} p_{n}$ the following algorithm returns a lower bound on the inversive threshold, $r(p)$ : 
Step 0. If $p=\epsilon$ return(0).

Step 1. While ( $\exists$ letter $c$ occurring at most once in each component of $p$ ) delete letter $c$ from $p$.

Step 2. If $p=\epsilon \operatorname{return}(1)$, else return $(\underline{r}(p))$.

In particular, $p$ has a nontrivial inversion if and only if Step 1 does not reduce $p$ to the empty string.

Theorem 10 gives a combinatorial characterization of patterns that have inversions and it is also very easy to implement. That there are patterns $p$ with $\underline{r}(p)<$ $r(p)<\bar{r}(p)$ can be seen by taking $p=a b a_{-} c b c_{-} b a c b$, for which $\underline{r}(p)=4 / 3$, $r\left(a b a_{-} c b c \_b a c b\right)=\sqrt{2}$ and $\bar{r}(p)=3 / 2$. The determination of $r(p)$ is a fascinating open problem, but one that appears difficult.

\subsection{Unavoidable Patterns}

In seeking to show that a pattern $p$ is unavoidable, one naturally investigates what kind of additional structure may be imposed on an $\omega$-word avoiding $p$. An $\omega$-word $w$ is recurrent if every finite subword of $w$ occurs infinitely often in $w$. If for each finite subword of a recurrent word $w$ there is a bound on the distance between consecutive occurrences of that subword, then we say that $w$ is recurrent with bounded gaps.

Theorem 11 Let $P$ be a set of patterns. If $P$ is $k$-avoidable in the abelian sense then there is an $\omega$-word on $k$ letters that avoids $P$ in the abelian sense and has the additional property of being recurrent with bounded gaps.

Proof For definiteness, let $\Sigma=\{1,2,3, \ldots, k\}$. Let

$$
\mathcal{S}=\left\{S \subset \Sigma^{*} \mid \exists w \in \Sigma^{\omega} w \not \models P \text { and } w \text { avoids subwords in } S\right\} .
$$

First notice that the set $\mathcal{S}$ is not empty. Since $P$ is $k$-avoidable, let $w \in \Sigma^{\omega}$ avoid $P$. Let $q$ be a pattern in $P$. It follows that $w$ avoids the word $1^{|q|}$ as a subword, since $1^{|q|} \models q$. Thus $\left\{1^{|q|}\right\} \in \mathcal{S}$.

We will show that the partial order $(\mathcal{S}, \subseteq)$ is chain complete. Let $\left\{S_{\alpha}\right\}_{\alpha \in I}$ be a chain in the partial order, and put $S=\bigcup_{\alpha} S_{\alpha}$. We claim that $S \in \mathcal{S}$. To see this let $S_{n} \subseteq S$ be the set of all strings in $S$ of length $\leq n$. Since $S_{n}$ is finite and $\left\{S_{\alpha}\right\}_{\alpha \in I}$ is a chain, we have $S_{n} \subset S_{\gamma}$ for some $\gamma \in I$. Since $S_{\gamma} \in \mathcal{S}$ there is a $u \in \Sigma^{\omega}$ that avoids $P$ in the abelian sense and avoids subwords in $S_{\gamma}$, hence $u$ avoids subwords in $S_{n}$. The prefix of length $n$ of $u, u_{n}$, also avoids $P$ in the abelian sense, and furthermore $u_{n}$ contains no string of $S$ as a substring. Each word in the set $\left\{u_{n}\right\}_{n \geq 1} \subseteq \Sigma^{*}$ therefore avoids $P$ and avoids subwords in $S$. By König's infinity lemma there is an $\omega$-word $v \in \Sigma^{\omega}$ with both of these properties, that is $v$ avoids $P$ and avoids subwords in $S$. Therefore $S \in \mathcal{S}$, establishing our claim and proving that $(\mathcal{S}, \subseteq)$ is chain complete. By Zorn's lemma, let $\hat{S} \in \mathcal{S}$ be a maximal element and let $w \in \Sigma^{*}$ avoid $P$ in the abelian sense and avoid subwords in $\hat{S}$. 
We claim that $w$ is recurrent. Otherwise, some subword $s$ occurs only finitely often in $w$. This means that some suffix $w^{\prime}$ of $w$ does not contain $s$ as a subword while avoiding $P$ in the abelian sense. Then $w^{\prime}$ avoids subwords in $\hat{S} \cup\{s\} \in \mathcal{S}$. Since $\hat{S} \cup\{s\}$ is strictly larger than $\hat{S}$, we have a contradiction. Therefore, $w$ avoids $P$ in the abelian sense and is recurrent.

If some subword $s$ of $w$ has arbitrarily long gaps between its occurrences in $w$, then $w$ has arbitrarily long subwords which avoid the subword $s$. By König's infinity lemma there is an $\omega$-word $w^{\prime}$ on $k$ letters that avoids $P$ and avoids subwords in $\hat{S} \cup\{s\} \in \mathcal{S}$, again contradicting the maximality of $\hat{S}$. Therefore $w$ is recurrent with bounded gaps. The proof is complete.

If $w$ is a recurrent word and a pattern $p$ occurs in $w$, then the substring of $w$ that gives the occurence of $p$ is repeated farther out in $w$, so we have two identical occurences of $p$ in $w$ separated by a non-empty substring. If 2 is some letter not in $p$ corresponding to the separating substring then $p 2 p$ occurs in $w$. Again, $p 2 p 3 p 2 p$ occurs in $w$, where 3 is a letter different from 2 and not a letter of $p$, and so on. A definition is in order:

Definition 12 Let $p$ be a word, none of whose letters is in $\mathbf{N}^{+}$. The $n$-th Zimin word seeded with $p$ is recursively defined by $Z_{1}(p)=p$ and $Z_{n}(p)=Z_{n}(p)(n) Z_{n}(p)$, $n \geq 2$.

Thus $Z_{4}(a b c)=a b c 2 a b c 3 a b c 2 a b c 4 a b c 2 a b c 3 a b c 2 a b c$, for example. Of course the ordinary Zimin words $Z_{n}=Z_{n}(1), n \geq 1$ are also seeded Zimin words. We now introduce functions $\rho, \lambda, \iota$ on words over the positive integers. Functions $\rho$ and $\lambda$ are morphisms generated by their action on integers:

$$
\begin{gathered}
\rho(i)=i+1 \\
\lambda(i)= \begin{cases}i-1, & i>1 \\
\epsilon, & i=1 .\end{cases}
\end{gathered}
$$

Function $\iota$ is given by

$$
\iota\left(w_{1} w_{2} w_{3} \cdots w_{n}\right)=1 w_{1} 1 w_{2} \cdots 1 w_{n} 1,
$$

where the $w_{i}$ are positive integers.

Remark 13 Let $\pi, \kappa$ be patterns over the positive integers with $\kappa=\kappa_{1} \cdots \kappa_{n}$, $\pi=$ $\pi^{\prime} K_{1} K_{2} K_{3} \cdots K_{n} \pi^{\prime \prime}$ where $K_{i} \sim K_{j}$ if $\kappa_{i}=\kappa_{j}$. We have

1. $\rho(\pi) \models \rho(\kappa)$

2. $\iota(\pi) \models \iota(\kappa)$ if neither of $\pi$ and $\kappa$ contains the letter 1

3. $\lambda(\pi) \models \lambda(\kappa)$ if $\kappa_{i}=1$ whenever $K_{i}=1$.

We also see that $Z_{n}=\iota\left(\rho\left(\lambda\left(Z_{n}\right)\right)\right)$ for all $n$. The following lemmas about Zimin words are readily established by induction: 
Lemma 14 If $A B C$ is a subword of $Z_{n}$ and $A \sim C$, then $B$ contains some integer greater than any integer in $A$.

Lemma 15 If $Z_{n} \models p$ for some $n \geq 1$ then $p$ is unavoidable in the abelian sense.

We now give an application of Theorem 11 that generalizes Lemma 15 above. Suppose that $Z_{n}(p) \models q$ for some $n$ and that $p$ is unavoidable on $k$ letters in the abelian sense. Then $q$ is also unavoidable in the abelian sense on $k$ letters. Otherwise by Theorem 11 there is a recurrent $\omega$-word $w$ on $k$ letters that avoids $q$, and then $w \models Z_{n}(p) \models q$, a contradiction. If $Z_{n}(p) \models q$ for some $n$ then we shall say that $p$ generates $q$ and write $p \rightarrow q$. Note that this relation is reflexive and transitive, but not symmetric. Let $\mathcal{U}_{k}$ be the set of patterns on $k$ letters that are unavoidable. We have proven the following:

Theorem 16 If $p \in \mathcal{U}_{r}$ and $p \rightarrow q$, then $q \in \mathcal{U}_{r}$.

An alternative, constructive way of looking at this result is the following: The polynomial $p_{r}(j)=\sum_{i=1}^{j}\left(\begin{array}{c}i+r-1 \\ r-1\end{array}\right)$ counts the number, up to abelian equivalence, of non-empty words of length $\leq j$ on $r$ letters. If $|w|=2 m+k, k \geq 0$ then there are $k(k+1) / 2$ factorizations of $w$ of the form $w=u X_{1} y X_{2} v$, where $\left|X_{1}\right|=\left|X_{2}\right|=m$, $y \neq \epsilon$, and $u, v$ may be empty. The function $k(n)=\left\lceil\frac{1}{2}(-1+\sqrt{8 n+9})\right\rceil$ then gives the smallest $k$ such that a string of length $2 m+k$ contains at least $n+1$ disjoint, nonadjacent pairs of strings of length $m$. Now suppose that $p \rightarrow q$ and $p \in \mathcal{U}_{r}$. Then there is a minimum length $\ell_{1}$ such that $p$ occurs in any string on $r$ letters of length $\ell_{1}$. We regard 2 occurrences of $p=a_{1} a_{2} \cdots a_{n}$ to be the same up to abelian equivalence if for each $i$ the block corresponding to $a_{i}$ in the first occurrence is a permutation of the block corresponding to $a_{i}$ in the second occurrence. Let $n_{1}$ be the number of ways $p$ can occur in a string of length $\ell_{1}$, up to abelian equivalence. Any string on $r$ letters of length $\ell_{2}=2 \ell_{1}+k\left(n_{1}\right)$ then contains an occurrence of $Z_{2}(p)$, and there are at most $n_{2}=n_{1} p_{r}\left(\ell_{2}-2|p|\right)$ different occurrences (up to abelian equivalence) of $Z_{2}(p)$ in such a string. In this way, by recursively defining $\ell_{j}=2 \ell_{j-1}+k\left(n_{j-1}\right)$ and $n_{j}=n_{j-1} p_{r}\left(\ell_{j}-2\left|Z_{j-1}(p)\right|\right)$ for $j \geq 2$, we see that $Z_{t}(p)$ occurs in any string on $r$ letters of length $\ell_{t}$. Since $p \rightarrow q$ we have $Z_{n}(p) \models q$ for some $n$, and it follows that $q$ occurs in any string on $r$ letters of length $\ell_{n}$.

The next two lemmas are devoted to proving that if $p \rightarrow q$ then $Z_{t}(p) \models q$, where $t$ is the number of distinct letters in $q$. Thus the problem of deciding if one pattern generates another one is decidable.

Lemma 17 Suppose that $q$ has $t$ distinct letters and $Z_{k} \models q$ for some $k$. Then $Z_{t} \models q$.

Proof Let $t, k$ be as above, and take the letters of $q$ to be positive integers. We will prove the theorem by induction on $t$. Suppose $t=1$. Without loss of generality, $q=1^{r}$ for some $r \geq 1$. However, $Z_{k} \models q$, and $Z_{k}$ is free of abelian squares. It follows that $q=1$, and $Z_{1} \models q$. The theorem thus holds for $t=1$. From now on, assume without loss of generality that both 1 and 2 are letters of $q$.

Now suppose that $t \geq 2$ and the theorem holds for $1,2, \ldots, t-1$. We will use induction on $k$. In the case where $k \leq t$ we have $Z_{t} \models Z_{k} \models q$, and the theorem 
holds. Suppose then that $k>t$. Fix an occurrence of $q=q_{1} q_{2} \cdots q_{n}$ in $Z_{k}$; say $Z_{k}=u R_{1} R_{2} \cdots R_{n} v$ where $R_{i} \sim R_{j}$ whenever $q_{i}=q_{j}$.

If $\lambda\left(R_{i}\right) \neq \epsilon$ for each $i$ then $\lambda\left(R_{i}\right) \sim \lambda\left(R_{j}\right)$ whenever $q_{i}=q_{j}$ and $Z_{k-1}=\lambda\left(Z_{k}\right) \models$ $\lambda\left(R_{1} R_{2} \cdots R_{n}\right) \models q$. By induction, $Z_{t} \models q$, and we are done.

Suppose then that $\lambda\left(R_{i_{0}}\right)=\epsilon$ for some $i_{0}$, i.e., $R_{i_{0}}=1$. We can suppose without loss of generality that $q_{i_{0}}=1$. We now wish to use the special structure of $Z_{k}$. Every second letter of $Z_{k}$ is a 1 . If for some $j$ we have $\left|R_{j}\right|$ even, then either we can write $R_{j}=1 Q_{j}$ for some word $Q_{j}$, or we can write $R_{j}=Q_{j} 1$ for some word $Q_{j}$. Further, if $R_{k} \sim R_{j}$, then either $R_{k}=1 Q_{k}$ or $R_{k}=Q_{k} 1$ where $Q_{k} \sim Q_{j}$. Analogous observations hold when $\left|R_{j}\right|$ is odd.

Form a new pattern $r=r_{1} r_{2} \cdots r_{n}$ as follows:

$$
r_{j}= \begin{cases}1, & \text { if } R_{j}=1 \\ 1 q_{j} 1, & \text { if } R_{j}=1 Q_{j} 1 \text { for some word } Q_{j} \neq \epsilon \\ 1 q_{j}, & \text { if } R_{j}=1 Q_{j} \text { for some word } Q_{j} \neq \epsilon \text { not ending in a } 1 \\ q_{j} 1, & \text { if } R_{j}=Q_{j} 1 \text { for some word } Q_{j} \neq \epsilon \text { not starting with } 1 \\ q_{j}, & \text { if } R_{j} \text { neither starts nor ends with a } 1 .\end{cases}
$$

We see that $r$ has at most $t$ distinct letters, one of which is a 1 , since $R_{i_{0}}=1$. By construction, $R_{1} R_{2} \cdots R_{n}$ is an occurrence of $r$, and $r \models q$. By Remark 13, $Z_{k-1}=$ $\lambda\left(Z_{k}\right) \models \lambda(r)$. Since 1 is a letter of $r, Z_{t-1} \models \lambda(r)$ by induction. It follows that $Z_{t}=\iota\left(\rho\left(Z_{t-1}\right)\right) \models \iota(\rho \lambda(r)) \models q$, so we get $Z_{t} \models q$. This completes the proof by induction.

We can prove a more general version of the above lemma.

Theorem 18 Suppose that $q$ has $t$ distinct letters and $Z_{k}(p) \models q$. Then $Z_{t}(p) \models q$.

Proof For definiteness we assume that the letters of $p$ and $q$ are from disjoint alphabets and that these two alphabets contain no integers. We may assume that $k>1$. Fix an occurrence of $q=q_{1} q_{2} \cdots q_{n}$ in $Z_{k}(p)$; say $Z_{k}(p)=\alpha R_{1} R_{2} \cdots R_{n} \beta$ where $R_{i} \sim R_{j}$ whenever $q_{i}=q_{j}$. Let $\delta$ be an operator that replaces substrings $p$ by the letter 1. Thus, for example, $\delta\left(Z_{k}(p)\right)=Z_{k}$. It is possible that substrings $p$ that overlap would render $\delta$ undefined, but we shall never apply $\delta$ in such a situation.

Case 1 Each $R_{i}$ contains at least one integer. Let $\epsilon$ be an operator that deletes the letters of $p$. Then $Z_{k-1} \models \lambda \epsilon\left(Z_{k}(p)\right)=\lambda \epsilon(\alpha) \lambda \epsilon\left(R_{1}\right) \lambda \epsilon\left(R_{2}\right) \cdots \lambda \epsilon\left(R_{n}\right) \lambda \epsilon(\beta)$. Further, the $\lambda \epsilon\left(R_{i}\right)$ are non-empty, and $\lambda \epsilon\left(R_{i}\right) \sim \lambda \epsilon\left(R_{j}\right)$ whenever $q_{i}=q_{j}$. Thus $Z_{k-1} \models q$. By Lemma 17 we find that $Z_{t} \models q$, where $t$ is the number of letters in $q$, hence $Z_{t}(p) \models Z_{t} \models q$, so $Z_{t}(p) \models q$, as desired.

Case 2 Each $R_{i}$ contains only letters from $p$. In this case, $Z_{t}(p) \models p \models q$, hence $Z_{t}(p) \models q$, as desired.

Case 3 Some $R_{i}$ contain only letters from $p$, but some $R_{i}$ contain integers. In this second case, as we read from left to right across the pattern $q$ we will encounter $n \geq 1$ 
letters $x_{1}, x_{2}, \ldots, x_{n}$ which correspond to those $R_{i}$ in $Z_{k}(p)$ that contain integers. Factor $q$ as $q=q_{1} x_{1} q_{2} x_{2} \cdots q_{n} x_{n} q_{n+1}$, and factor $Z_{k}(p)$ as $Z_{k}(p)=\alpha Q_{1} X_{1} Q_{2} X_{2} \cdots$ $Q_{n} X_{n} Q_{n+1} \beta$ where $X_{i} \sim X_{j}$ whenever $x_{i}=x_{j}$ and the $q_{i}$ are sub-patterns of $q$ whose occurrences on the $Q_{i}$ are compatible. None of the letters $x_{j}$ appears in any $q_{i}$. Some of the $q_{i}$ may be empty, but under our assumption at least one of them is not. We factor each string $X_{i}$ as $X_{i}=u_{i} X_{i}^{\prime} v_{i}, i=1,2, \ldots, n$ where $X_{i}^{\prime}$ begins with the first integer in $X_{i}$ and ends with the last integer in $X_{i}$. One or both of $u_{i}, v_{i}$ may be empty, but if $x_{i}=x_{j}$ then $u_{i} v_{i} \sim u_{j} v_{j}$. Putting in the factorizations of the $X_{i}$ we have

$$
\begin{aligned}
Z_{k}(p) & =\alpha Q_{1}\left(u_{1} X_{1}^{\prime} v_{1}\right) Q_{2}\left(u_{2} X_{2}^{\prime} v_{2}\right) \cdots Q_{n}\left(u_{n} X_{n}^{\prime} v_{n}\right) Q_{n+1} \beta \\
& =\alpha^{\prime}\left(v_{0} Q_{1} u_{1}\right) X_{1}^{\prime}\left(v_{1} Q_{2} u_{2}\right) X_{2}^{\prime} \cdots\left(v_{n-1} Q_{n} u_{n}\right) X_{n}^{\prime}\left(v_{n} Q_{n+1} u_{n+1}\right) \beta^{\prime} \\
& =\alpha^{\prime} p X_{1}^{\prime} p X_{2}^{\prime} p \cdots p X_{n}^{\prime} p \beta^{\prime}
\end{aligned}
$$

where the strings $v_{0}, u_{n+1}$ are borrowed from $\alpha$ and $\beta$ respectively to complete the formation of the interspersing $p$ 's.

The above factorization shows that $Z_{k}=\delta\left(Z_{k}(p)\right) \models 1 x_{1} 1 x_{2} 1 \cdots 1 x_{n} 1=q^{\prime}$ (recall the definition of the $\delta$ operator after Theorem 18). By Lemma 17 we have $Z_{t^{\prime}} \models q^{\prime}$, where $t^{\prime}$ is the number of letters in $q^{\prime}$.

Claim 19 We can find an occurrence of $q^{\prime}$ in $Z_{t^{\prime}}$ such that 1 in $q^{\prime}$ corresponds to 1 in $Z_{t^{\prime}}$.

Proof of Claim Write $Z_{t^{\prime}}$ as $U A_{1} Y_{1} A_{2} Y_{2} \cdots A_{n} Y_{n} A_{n+1} V$ where $A_{i} \sim A_{j}$ for all $i$, $j$, while $Y_{i} \sim Y_{j}$ if $x_{i}=x_{j}$. By Lemma 14, each $Y_{i}$ contains an integer higher than every integer in $A_{1}$. If $A_{1}=1$, then we are done. Otherwise, none of the $A_{i}$ or $Y_{i}$ are 1 , so by Remark 13,

$$
\begin{aligned}
Z_{t-1} & \models \lambda\left(Z_{t^{\prime}}\right) \\
& =\lambda\left(U A_{1} Y_{1} A_{2} Y_{2} \cdots A_{n} Y_{n} A_{n+1} V\right) \\
& \models \lambda\left(A_{1}\right) \lambda\left(Y_{1}\right) \lambda\left(A_{2}\right) \lambda\left(Y_{2}\right) \cdots \lambda\left(A_{n}\right) \lambda\left(Y_{n}\right) \lambda\left(A_{n+1}\right) \\
& \models q^{\prime}
\end{aligned}
$$

and the result can be established by induction.

Say then that $Z_{t^{\prime}}=\alpha 1 Y_{1} 1 Y_{2} 1 \cdots 1 Y_{n} 1 \beta$ where $Y_{i} \sim Y_{j}$ whenever $x_{i}=x_{j}$. This yields an occurrence of $q^{\prime}$ in $Z_{t^{\prime}}(p)=\alpha^{\prime} p Y_{1} p Y_{2} p \cdots p Y_{n} p \beta^{\prime}$ where 1 in $q^{\prime}$ corresponds to $p$ in $Z_{t^{\prime}}(p)$. Since $p=v_{i-1} Q_{i} u_{i}$ for $i=1,2, \ldots, n+1$ we have

$$
\begin{aligned}
Z_{t^{\prime}}(p) & =\alpha^{\prime}\left(v_{0} Q_{1} u_{1}\right) Y_{1}\left(v_{1} Q_{2} u_{2}\right) X_{2}\left(v_{2} Q_{3} u_{3}\right) \cdots\left(v_{n-1} Q_{n} u_{n}\right) Y_{n}\left(v_{n} Q_{n+1} u_{n+1}\right) \beta^{\prime} \\
& =\alpha^{\prime \prime} Q_{1}\left(u_{1} Y_{1} v_{1}\right) Q_{2}\left(u_{2} X_{2} v_{2}\right) Q_{3} \cdots Q_{n}\left(u_{n} Y_{n} v_{n}\right) Q_{n+1} \beta^{\prime \prime} \\
& =\alpha^{\prime \prime} Q_{1} Y_{1}^{\prime} Q_{2} Y_{2}^{\prime} Q_{3} \cdots Q_{n} Y_{n}^{\prime} Q_{n+1} \beta^{\prime \prime}
\end{aligned}
$$


where $Y_{i}^{\prime}=u_{i} Y_{i} v_{i}$ for $i=1,2, \ldots, n$. Since $u_{i} v_{i} \sim u_{j} v_{j}$ and $Y_{i} \sim Y_{j}$ whenever $x_{i}=x_{j}$ we have $Y_{i}^{\prime} \sim Y_{j}^{\prime}$ whenever $x_{i}=x_{j}$. Furthermore, for each $i$ we may construct an occurrence of $q_{i}$ on the string $Q_{i}$ in such a way that all of these occurrences are compatible, simply by copying from the initial occurrence of $q$ in $Z_{k}(p)$. We have now constructed an occurrence of $q$ in $Z_{t^{\prime}}(p)$.

Recall that at least one $q_{i}$ is not empty, and the letters comprising the $q_{i}$ are disjoint from the letters $x_{1}, x_{2}, \ldots, x_{n}$. Thus in passing from $q=q_{1} x_{1} q_{2} \cdots q_{n} x_{n} q_{n+1}$ to $x_{1} x_{2} \cdots x_{n}$ at least one letter is lost, and then by forming $q^{\prime}=1 x_{1} 1 x_{2} 1 \cdots 1 x_{n} 1$ one letter is gained, so $t^{\prime} \leq t$. Finally we have $Z_{t}(p) \models Z_{t^{\prime}}(p) \models q$, hence $Z_{t}(p) \models q$, as desired.

Combining Theorem 16 with Theorem 18 we have:

Corollary 20 If $p \in \mathcal{U}_{r}$ and $p \rightarrow q$, then $q$ occurs in the abelian sense in any string on $r$ letters of length $\ell_{t}$, where $\ell_{t}$ is defined in the proof of Theorem 16 and $t$ is the number of distinct letters in $q$.

We now describe an idea that is useful in reducing the amount of backtracking needed to show that a pattern is unavoidable in the abelian sense on a given number of letters. If $p$ and $q$ do not contain the letter $x$ then one may argue as in the proof of Theorem 16 that $p_{-} q \in \mathcal{U}_{k}$ implies $p x q \in \mathcal{U}_{k}$, and hence both of these patterns require the same number of letters.

Lemma 21 If $p$ and $q$ are patterns not containing the letter $x$ then $p_{-} q \in \mathcal{U}_{r}$ if and only if $p x q \in \mathcal{U}_{r}$.

Proof If $p x q \in \mathcal{U}_{r}$ then clearly $p_{-} q \in \mathcal{U}_{r}$. Suppose that $p_{-} q \in \mathcal{U}_{r}$ but that $p x q \notin \mathcal{U}_{r}$. By Theorem 11 there is a recurrent $\omega$-word $w$ that avoids $p x q$. However, $p-q \in \mathcal{U}_{r}$ so $p_{-} q$ occurs in the abelian sense in $w$. The recurrence of $w$ gives us two identical occurrences of $p_{-} q$, which together yield an abelian occurrence of $p x q$, a contradiction. Therefore $p_{-} q \in \mathcal{U}_{r}$ implies $p x q \in \mathcal{U}_{r}$ and the proof is complete.

To illustrate how Lemma 21 can be used consider the problem of showing that $p=01021 \in U_{2}$. The string 001100100 avoids $p$ and has length 9 , but $p_{1}=010 \_1$ occurs in any string of length 5 on 2 letters. Indeed, occurrences of $p_{1}$ are equivalent to occurrences of $p_{2}=010$, which is equivalent to $p_{3}=0 \_0$, which is the same as $p_{4}=0$, and the latter is certainly unavoidable.

\section{Patterns on 3 and 4 Letters}

\subsection{The Case of 3 Letters}

By Lemma 7, any pattern on 3 letters of length 8 or more is avoidable in the abelian sense. By [11] abelian squares are avoidable, hence so is any pattern encountering an abelian square. We need therefore only examine patterns of length at most 7 that are free of abelian squares. These are generated in Figure 3.1, where we assume that all patterns start with 01. 


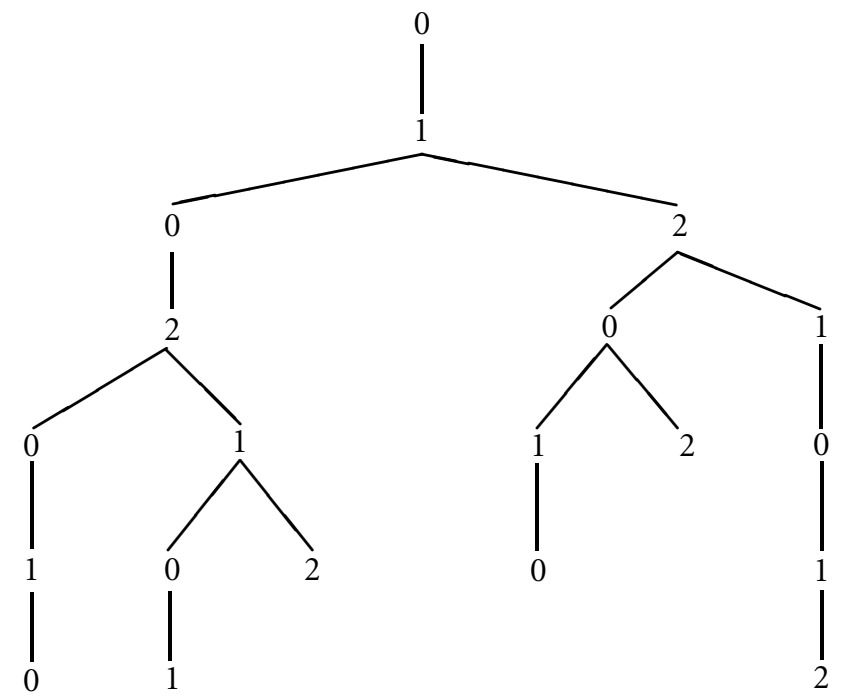

Figure 1: Words on $\{0,1,2\}$ starting with 01 and avoiding abelian squares.

We find that the patterns 0102010,012010 and 01202 all occur in the abelian sense in $Z_{3}$, while on the other hand the patterns 0102101,010212 and 0121012 have inversions, as they force $x^{\frac{3}{2}+}, x^{\frac{4}{3}+}$ and $x^{\frac{5}{4}+}$ respectively. It follows from our analysis that a pattern $p$ on 3 letters is avoidable in the abelian sense if and only if $Z_{3} \not \models p$.

\subsection{The Case of Four Letters}

First of all, by Lemma 7 we need only consider patterns on 4 letters of length $\leq 15$. Of course, this still leaves over $4^{15}$ possibilities, so further reductions are necessary. By permuting letters, we need only consider patterns that begin with $p_{1}=0102$, $p_{2}=0120, p_{3}=0121$ or $p_{4}=0123$. Using an implementation of the algorithm in Theorem 10 we reject an extension of a $p_{i}$ that has a nontrivial inversion, since such a pattern is abelian avoidable.

As the length of a pattern increases the chances of it having an inversion also increases, and a short pattern is more likely to be unavoidable. It turns out to be the case that the number of patterns of a given length that do not fall under the purview of Lemma 15 or Theorem 10 first increases and then decreases in the manner of a normal distribution. There are 132 such patterns in total if we regard two patterns as the same when one can be obtained from the other by permuting letters and/or reversing. These 132 pattern make up the $B$-list (" $B$ " for "bad", because for these patterns we cannot decide abelian avoidability). How these patterns are distributed according to length is shown in Table 3.2.

If we partially order the $B$-list by the relation $p \leq q$ if and only if $q \models p$ or $q^{R} \models p$ then we find that there are exactly six minimal elements, which necessarily includes all four of the patterns of length 8 in the $B$-list. Each of the 132 patterns essentially encounters one of the six patterns given in Table 3.2. If we could show those six 


\begin{tabular}{|c|c|c|c|c|c|c|c|c|c|c|}
\hline length & $\leq 7$ & 8 & 9 & 10 & 11 & 12 & 13 & 14 & 15 & total \\
\hline \# of patterns & 0 & 4 & 18 & 36 & 39 & 24 & 9 & 2 & 0 & 132 \\
\hline
\end{tabular}

Table 3: Frequency distribution of the B-list.

\begin{tabular}{|l|l|}
\hline$b_{1}$ & 01020312 \\
$b_{2}$ & 01020321 \\
$b_{3}$ & 01021303 \\
$b_{4}$ & 01023031 \\
$b_{5}$ & 010203013 \\
$b_{6}$ & 010213020 \\
\hline
\end{tabular}

Table 4: The six minimal patterns in the B-list.

patterns are abelian avoidable then it would follow that all patterns on 4 letters which do not encounter $Z_{4}$ are avoidable.

This last list of six we shall refer to as the short B-list.

\section{Analysis of the Short B-List}

In this section we will analyse the patterns of the short $B$-list and give some of their properties. The main outcome of our exploration is a lower bound on the number of letters needed to avoid these patterns and a further reduction of the $B$-list due to symmetries present in the patterns.

\subsection{Symmetries}

Let $p^{R}$ denote the reverse of the pattern $p$, for example $a b c b a a c^{R}=c a a b c b a$. If $w=p x q$, where $p$ and $q$ are words not containing the letter $x$ then $p x q^{R}$ is a conjugate of $w$. The word $w=$ abcadcabeabaa then has 2 conjugates: $w_{1}=$ abcadaabaebac and $w_{2}=$ abcadcabeaaba. The word $w$ is a palindrome if $w=w^{R}$.

Lemma 22 If $p, q$ do not contain the letter $x$ and $p$ is a palindrome then $f=p x q \in$ $\mathcal{U}_{k}$ if and only if $\bar{f}=p x q^{R} \in \mathcal{U}_{k}$.

Proof By Lemma $21 f \in \mathcal{U}_{k}$ if and only if $p_{-} q \in \mathcal{U}_{k}$, which is equivalent to $q^{R}-p=$ $q^{R}-p^{R}=\left(p_{-} q\right)^{R} \in \mathcal{U}_{k}$ which is equivalent to $\bar{f}=p x q^{R} \in \mathcal{U}_{k}$ by Lemma 21 .

Note that if Conjecture 2 is true then it would follow that $p x q$ is unavoidable if and only if $p x q^{R}$ is unavoidable, $p, x$ and $q$ as in the hypotheses of Lemma 22. However, the statement of Lemma 22 is more specific since it involves $k$, the alphabet size. It is natural to make the following 
Conjecture 23 If $p, q$ do not contain the letter $x$ then $f=p x q \in \mathcal{U}_{k}$ if and only if $\bar{f}=p x q^{R} \in \mathcal{U}_{k}$.

Given $k$, Lemma 22 says that the patterns 01021303 and 01023031 are both $k$ unavoidable or both $k$-avoidable in the abelian sense. This is also the case for 010203013 and 010213020 , since $010213020^{R}$ is a conjugate of 010203013 with a palindromic prefix. This further reduces the short $B$-list to (say) the short, short $B$-list consisting of 01020312, 01020321, 01021303 and 010203013.

\subsection{Minimal Lengths and Minimal Alphabets}

In order to apply some of our lemmas to the patterns in the short, short $B$-list we need to find some patterns that generate these. We define the spectrum of $p$, denoted $\operatorname{Spec}(p)$, as the set of all patterns that generate $p$. Since the property of one pattern generating another is transitive, we seek to find minimal elements of the spectrum of a pattern. Thus if $p \rightarrow q$ and $f \models p$ then $f \rightarrow q$, and we will not use $f$ in our computations.

We find that $Z_{4}(00) \models 01021303,010203013$. Also, $Z_{3}(00) \models 01020312$, 01020321. Thus 00 is in the spectra of these patterns. By the computation we did in Figure 3.1, all strings on 3 letters of length 8 or more contain an abelian square, so we look for shorter strings in the spectra. The only patterns in the tree of Figure 3.1 that do not occur in $Z_{3}$ are $g_{1}=0102101, g_{2}=0121012$ and $g_{3}=010212$. These therefore are the only patterns that can generate patterns on the short $B$-list. We find that 010212 does not generate any $b_{i}$, but that 0102101,0121012 are in the spectra of all $b_{i}$. Also, no pattern on 4 letters of length 7 or less can generate any $b_{i}$, since these patterns either occur in $Z_{4}$ or encounter one of the $g_{i}$. We find that no patterns in $\left\{b_{1}, b_{2}, b_{3}, b_{5}\right\}$ can generate any other pattern in this set. We have

Lemma 24 The patterns $b_{1}$ and $b_{2}$ occur in the abelian sense in any string of length 214 over a 3 letter alphabet, the patterns $b_{3}$ and $b_{5}$ occur in any string of length 208143 over a 3 letter alphabet.

Proof Apply Lemma 20 with $r=3, p=00, \ell_{1}=8$, and $n_{1}=13$ to obtain $\ell_{3}=214$ and $\ell_{4}=208143$.

Lemma 25 All patterns in the short B-list are unavoidable on 4 letters.

Proof Utilizing a back tracking algorithm we find that the longest word on 4 letters that begins with 0102 and avoids in the abelian sense both 0102101 and 0121012 has length 54. Next we find that a longest string on 4 letters that begins with 0120 and avoids 0102101,0121012 and $a b a$ as a substring has length 29 . Finally we see that the longest string on 4 letters avoiding our patterns and with each four consecutive letters distinct has length 7 . This proves that 0102101 and 0121012 are not simultaneously avoidable on 4 letters, hence all patterns in the short $B$-list are unavoidable on four letters since they each have 0102101 and 0121012 in their spectra. 
This last result again underscores the difference between the abelian case and the ordinary case, as no pattern is known which is avoidable in the ordinary sense yet requires more than 4 letters to be avoided.

\section{References}

[1] Kirby A. Baker, George F. McNulty and Walter Taylor, Growth problems for avoidable words. Theoret. Comput. Sci. (3) 69(1989), 319-345. MR 91f:68109.

[2] Dwight R. Bean, Andrzej Ehrenfeucht and George McNulty, Avoidable Patterns in Strings of Symbols. Pacific J. Math. 85(1979), 261-294; MR 81i:20075.

[3] Jean Berstel, Axel Thue's papers on repetitions in words: a translation.

[4] Stanley Burris and Evelyn Nelson, Embedding the dual of $\Pi_{\infty}$ in the lattice of equational classes of semigroups. Algebra Universalis 1(1971/72), 248-253. MR 45 \#5257.

[5] Arturo Carpi, On abelian squares and substitutions. Theoret. Comput. Sci. (1) 218(1999), 61-81.

[6] Julien Cassaigne, Motifs évitables et régularités dans les mots. Thèse de doctorat, L.I.T.P., Université Paris 6, 1994.

[7] J. Cassaigne and J. Currie, Words strongly avoiding fractional powers. European J. Combin., to appear.

[8] James D. Currie, Open problems in pattern avoidance. Amer. Math. Monthly 100(1993), 790-793.

[9] F. M. Dekking, Strongly non-repetitive sequences and progression-free sets. J. Combin Theory Ser. A 27(1979), 181-185. MR 81b:05027.

[10] Paul Erdős, Some unsolved problems. Magyar Tud. Akad. Mat. Kutato. Int. Kozl. 6(1961), 221-254.

[11] A. A. Evdomikov, Strongly asymmetric sequences generated by a finite number of symbols. Dokl. Akad. Nauk. SSSR 179(1968), 1268-1271; Soviet Math. Dokl. 9(1968), 536-539.

[12] Veikko Keränen, Abelian squares are avoidable on 4 letters. In: Automata, Languages and Programming, Lecture Notes in Comput. Sci. 623, Springer-Verlag, 1992, 41-52.

[13] Marston Morse and Gustav A. Hedlund, Symbolic dynamics I, II. Amer. J. Math. 60(1938), 815-866; 62(1940), 1-42. MR 1, 123d.

[14] P. S. Novikov and S. I. Adjan, Infinite periodic groups I, II, III. Izv. Akad. Nauk. SSSR Ser. Mat. 32(1968), 212-244; 251-524; 709-731. MR 39 \#1532a-c.

[15] P. A. B. Pleasants, Non-repetitive sequences. Proc. Cambridge Philos. Soc. 68(1970), 267-274. MR 42 $\# 85$.

[16] L. N. Shevrin and M. V. Volkov, Identities of semigroups. Math. USSR Izv. (11) 29(1985), 3-47.

[17] Axel Thue, Über unendliche Zeichenreihen. Norske Vid. Selsk. Skr. I. Mat.-Nat. Kl. Christiana (1906), Nr. 7.

[18] A. Zimin, Blocking sets of terms. Mat. Sb. (N.S.) 119 (161)(1982), 363-375, 447; Math. USSR-Sb. 47(1984), 353-364.

Department of Mathematics and Statistics

University of Winnipeg

Winnipeg, Manitoba

R3B $2 E 9$ 